\title{
Fuzzy Energy Management for Photovoltaic Water Pumping System
}

\author{
Imene Yahyaoui \\ Dept. Systems Engineering and \\ Automatic \\ Industrial Eng. School \\ Valladolid, Spain
}

\author{
Maher Chaabene \\ Dept. Electric Engineering \\ National School of Electronics \\ and Telecommunications \\ Sfax, Tunisia
}

\author{
Fernando Tadeo \\ Dept. Systems Engineering and \\ Automatic \\ Industrial Eng. School \\ Valladolid, Spain
}

\begin{abstract}
A Fuzzy energy management algorithm for a photovoltaic water pumping installation is presented and validated experimentally, using a plant composed of photovoltaic panels, battery banks, DC/AC converters, relays and a water pump. Considering many criteria related to the battery safe operation, fulfilling the water volume needed by the crops and ensuring a continuous function of the pump, the algorithm decides the switching of the relays, which link the installation' components. The algorithm is evaluated for a specific case study: tomato irrigation in Tunisia. Obtained results confirm that the irrigation water demand is covered during the season with a minimum use of the battery.
\end{abstract}

\section{General Terms}

Fuzzy logic, Photovoltaic Energy management.

\section{Keywords}

Photovoltaic energy, fuzzy logic, management, water, pumping.

\section{INTRODUCTION}

Currently, there exist many remote agriculture sites without access to an electricity grid. Extending the main utility grids to these remote locations is possible, but not cost effective [12]. Hence, electricity is usually provided to these remote areas through stand-alone power systems. For agriculture applications, diesel power plants are generally used for water pumping, in such distant areas, since they are reliable, easily available and easy to use. However, experience has demonstrated that there are significant limitations associated with this method of power generation, such as the high cost to run and maintain the diesel engine, and in particular the diesel transportation high cost [3] and the environmental pollution [4]. Consequently, water pumping installations based on renewable energies are increasingly deployed in remote areas.

In fact, several works have focused on photovoltaic irrigation systems. For instance, [5] found that photovoltaic energy is more efficient than diesel engines in water pumping installations, in terms of economic and environmental arguments. Moreover, [6] compared the use of photovoltaic and wind energy for water pumping and found that, in well sunny sites, photovoltaic energy is more efficient than wind energy for water pumping.

Hence, many researches have been developed to enhance the efficiency of photovoltaic based installations destined for water pumping [7, 8]. For example, [9] focused in determining the optimum sizing for the water pumping photovoltaic installations. Others works concentrated in water pump modeling and control [10] or in the system modeling and simulation of photovoltaic generators and converters used to feed asynchronous machines actuating a centrifugal pump $[11,12]$. Researchers also established some management algorithms to the optimum use of photovoltaic energy [13], using intelligent tools.

In this sense, Fuzzy logic has been used for the energy management in photovoltaic based systems [13, 14]. Indeed, in autonomous photovoltaic installations, which are composed of photovoltaic panels, a battery bank and converters, fuzzy logic has been used to optimize the photovoltaic energy generated, to guarantee the installation' autonomy, ensure a safe operation for the battery' bank and supply the loads [13, 15]. Moreover, this tool has been used in renewable based installations, which supply controllable loads $[14,16]$ and constant critical and non-critical loads [17]. Furthermore, owing to its simplicity in implementation, Fuzzy logic has been used in simpler applications, such as agriculture. For instance, it has been used to decide the irrigation schedule and nutrient injection, depending on the climatic parameters (solar radiation, humidity, etc.) [18]. This tool has also been used for controlling the internal climatic variables in greenhouses [19] and for the energy management of water pumping installations, in which optimum algorithms called Fuzzy Management Algorithms (FMA) have been used to maximize the pumped water $[13,15,20]$.

The efficiency of this tool in various applications is given by its ease of use. In fact, in some problems, it is complicated to give an exact value for criteria, such as in case of energy management $[13,14]$. Thus, fuzzy logic is considered a good method for these types of problems, since it gives the possibility to describe system behaviors, or decide control decisions using linguistic rules $[20,21]$. Moreover, based on the expert knowledge, the fuzzy rules are written in a simple linguistic manner that describes the adopted approach in taking control decision. Mamdani-type fuzzy logic is used within the management algorithm, as it is simple to learn for operators with little technical training $[15,22]$ and can be implemented using standard components, such as Programmable Industrial Controllers [23]. Hence, when energy management is based on case study, it is obvious to choose fuzzy logic as a control tool.

This paper presents a continuation of previous published works which studied the pumping management algorithm for 


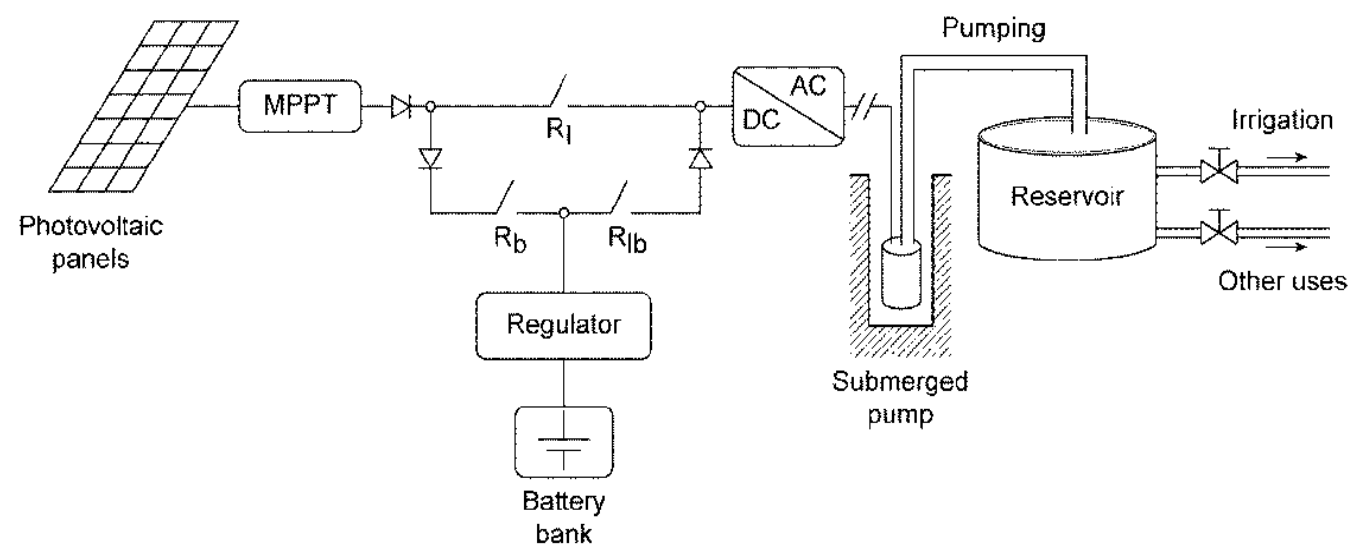

Fig. 1. Scheme of the off-grid photovoltaic irrigation system

irrigating tomatoes [24]. The present paper focuses on the experimental validation of an energy management algorithm of the autonomous photovoltaic irrigation plant, presented in Fig. 1. Photovoltaic panels are coupled to a lead-acid battery bank to ensure the energy availability, even if the irradiation is low. Based on some previous proposals [15, 16, 21], a general fuzzy management algorithm (FMA) is proposed and validated here. Using meteorological measurements, namely the solar radiation and the ambient temperature, and the water volume needed by the plants, the algorithm decides the switching of the relays, which link the installation's elements, to ensure a continuous pump supply and a safe battery bank operation. The connection or disconnection of the components is decided by fuzzy rules, which depend on the measured Photovoltaic Panel Generation, the depth of discharge of the battery bank, the water level in the reservoir and the water flow (Fig.2).

The detailed fuzzy management algorithm is presented in section 2, where the management strategy and the algorithm's execution are explained in depth. Obtained experimental results are presented and discussed in section 3. Finally, section 4 gives a conclusion.

\section{ENERGY MANAGEMENT \\ ALGORITHM}

The energy management algorithm aims to optimize the use of the electrical energy produced from a photovoltaic system composed of a photovoltaic generator and a battery bank, which supply a water pump (Fig.1). The fuzzy management algorithm (FMA) principle is explained here [24, 25].

\subsection{Management Strategy}

A management algorithm is established to meet the crops' water need through the control of the relays, which link the system' components (Fig.2). Hence, the fuzzy algorithm is to decide the interconnection time of the system elements using only the expert knowledge [13, 16, 24].

In fact, the energy management algorithm is based on four steps: the knowledge base of the expert, the fuzzification, the inference diagram and the defuzzification [14, 20, 24]. The interconnection time decision of the system components is made by means of fuzzy rules that fulfill the following objectives:

O1) Minimize the use of the battery bank.

O2) Provide the required irrigation when needed, by storing water in the reservoir.
O3) Protect the batteries against the excessive charge and discharge, by disconnecting them, respectively, from PVs and the pump when they are not used.

O4) Ensure a continuous power supply, especially during weather changes.

As photovoltaic power is used to supply the pump and the batteries, the water pumping is normally performed during the daylight to minimize the battery use. This facilitates to keep the depth of discharge of the battery bank ( $d o d$ ) between two fixed values $\operatorname{dod}_{\min }$ and $\operatorname{dod}_{\max }$, for a continuous pump operation (that stops when the tank is full or the battery discharged).

The management algorithm decides the switching times of the three relays $R_{b}, R_{l}$ and $R_{l b}$, that connect the photovoltaic system elements (Fig. 3). Hence, it is necessary to establish some criteria that define the algorithm efficiency. These criteria are related to:

i. The photovoltaic energy produced by the panel $P_{p v}$.

ii. The battery depth of discharge $\mathrm{dod}$.

iii. The water volume in the reservoir $L$.

The management criteria are defined as follows:

a) When the reservoir contains enough water, store the excess of photovoltaic energy in the batteries.

b) Maintain a high water level in the reservoir to guarantee the water volume needed for the crop irrigation.

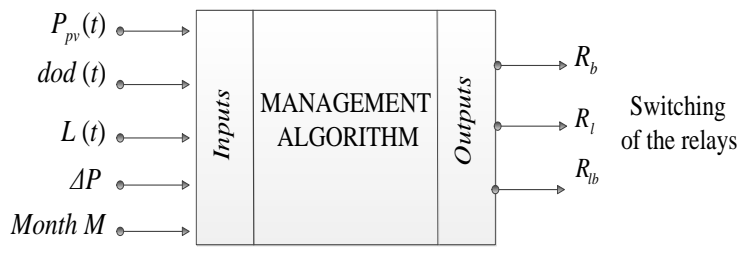

Fig. 2. The structure of the proposed energy management algorithm

c) Ensure a depth of discharge $\operatorname{dod}$ less than $\operatorname{dod}_{\max }$ to protect the battery against deep discharge, and greater than $\operatorname{dod}_{\text {min }}$ to protect it from excessive charge.

d) Ensure a margin of $10 \%$ of the photovoltaic power: the pump can be connected only to the panel if the measured 
photovoltaic power is $10 \%$ higher than the required power by the pump, to guarantee a continuous power supply for the pump.

During the day, the instantaneous power $P_{\text {pump }}$ verifies that:

$$
P_{p u m p}=P_{p v}+\bar{P}_{B a t}
$$

and the current absorbed by the load $I_{\text {pump }}$ is just:

$$
I_{\text {pump }}=I_{p v}+\bar{I}_{\text {Bat }}
$$

According to the fourth criterion, the panel supplies the load alone if it can provide at least $110 \%$ of the demand. This criterion is to guarantee the stability of the supply. Thus:

$$
I_{p v} \geq 1.1 I_{p u m p}
$$

The proposed energy management algorithm is performed via two steps: The first step consists in the acquisition of the climate-related installation site parameters, which allows the photovoltaic power $P_{p v}$ to be estimated. The second step is to deduce the load connection times and duration to the power sources (Fig. 3).

\subsection{Switching Mode}

To achieve our objectives listed above, six operating modes for the three relays $R_{b}, R_{l}$ and $R_{l b}$ have been defined:

1/ At night, in normal conditions, the volume in the tank is full, so all the switches are off (mode 1). This mode is maintained during the irrigation period where the tank volume decreases.

2/ In the early hours of the morning, mode 2 is possible since the battery and the panels provide the pump with electric power to ensure the water pumping. In this case, the relays $R_{l}$ and $R_{l b}$ are on.

3/ The third mode (mode 3) consists in pumping water and charging the battery with the energy in excess. In this case, the relays $R_{l}$ and $R_{b}$ are on.

4/ When the reservoir is full, the photovoltaic energy produced by the panel is used in total to charge the battery. This is possible when the battery is discharged and it corresponds to mode 4 .

$5 /$ The relay $R_{l}$ is switched on during the fifth mode (mode 5 ), to allow the pump supplying. This is possible when the panel produces the sufficient power to the pump with an excess of $10 \%$.

6/ During mode 6, only the relay $R_{l b}$ is switched on. This mode is possible during the night when the water volume in the reservoir is less than the volume needed to irrigate the crops for the corresponding month.

\subsection{Fuzzy Management Algorithm}

Fuzzy decisions are built upon four steps [15, 16, 21, 25]: the creation of the knowledge base, the fuzzification, the inference diagram, and the defuzzification. These four steps are now presented in detail.

\subsubsection{Knowledge Base}

The knowledge base is generated on the basis of specifications analysis:

Photovoltaic power $P_{p v}$

The photovoltaic generated power $P_{p v}$ is periodically measured and then partitioned in three fuzzy sets that cover the interval $\left[0, X=\left[0, P_{p v \max }\right]\right.$ at low, medium and high generation levels, respectively:

$$
\forall x \in X, \mu_{L}(x)+\mu_{M}(x)+\mu_{H}(x)=1
$$

where $\mu_{L}(x), \mu_{M}(x)$ and $\mu_{H}(x)$ are, respectively, the low, medium and high membership functions at the measured power level $x$.

\section{Battery dod}

It is composed of three fuzzy sets that cover the interval $D=\left[0, \operatorname{dod}_{\max }\right]$ at low, medium and high production levels, respectively, and verify:

$$
\forall d \in D, \mu_{d L}(d)+\mu_{d M}(d)+\mu_{d H}(d)=1
$$

where $\mu_{d L}(d), \mu_{d M}(d)$ and $\mu_{d H}(d)$ are, respectively, the low, medium and high membership functions of $\operatorname{dod} d$.

\section{Stored water $v$}

The third partition is composed of three fuzzy sets in the interval $V=\left[0, V_{\max }\right]$ which verify:

$$
\forall v \in V, \mu_{v L}(v)+\mu_{v M}(v)+\mu_{v H}(v)=1
$$

where $\mu_{v L}(v), \mu_{v M}(v)$ and $\mu_{v H}(v)$ are, respectively, the membership functions of $v$.

As the definition of low, medium and high depends on the use of the auxiliary sets, the following fuzzy variables are defined:

\section{- $\quad$ Month $M$}

This partition is composed of as many fuzzy sets as months, given by the interval $M=\left(m_{1}, m_{2}, \ldots, m_{t}\right)$ and verify:

$$
\forall m \in M, \mu_{m_{1}}(m)+\mu_{m_{2}}(m)+\ldots+\mu_{m_{t}}(m)=1
$$

Where $\mu_{m_{i}}(m)$ are the membership functions corresponding to the month $m$.

- Water level $L$

This partition is composed of as many fuzzy sets as months, denoted by the interval $L=\left(l_{1}, l_{2}, \ldots, l_{t}\right)$. The interval of the 


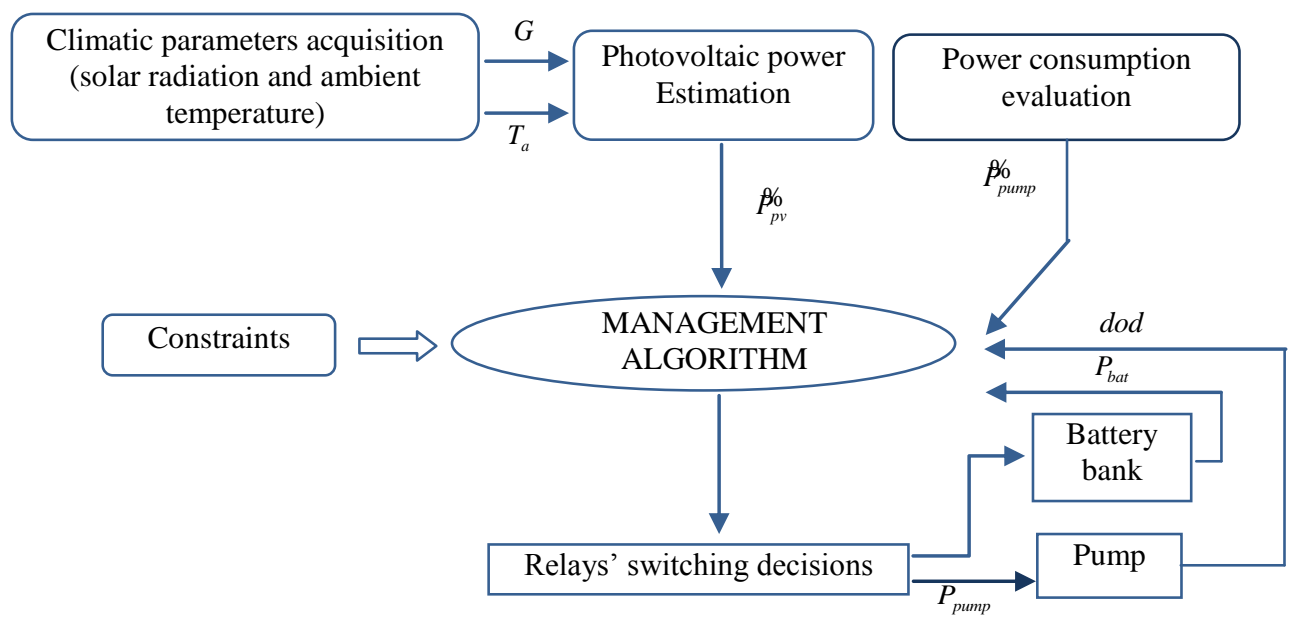

Fig. 3. Energy management strategy

possible water $L=\left[0, L_{\max }\right]$ is covered by these sets and verify:

$$
\forall l \in L, \mu_{l_{1}}(l)+\mu_{l_{2}}(l)+\ldots+\mu_{l_{t}}(l)=1
$$

where $\mu_{l_{i}}(l)$ is the membership function corresponding to $l_{i}$ evaluated at $l$.

\section{Power difference $\Delta P$}

This partition is composed of two fuzzy sets $F=\left(f_{1}, f_{2}\right)$ and verify:

$$
\forall f \in F, \mu_{f_{1}}(f)+\mu_{f_{2}}(f)=1
$$

where $\mu_{f_{e}}(f)$ is the membership function corresponding to $f_{e}$ evaluated at $f$.

\section{Relays $R_{l}, R_{b}, R_{l b}$}

To decide the switching of the relays $R_{l}, R_{b}, R_{l b}$, depending on the fuzzy variables $x, d$ and $v$, two fuzzy sets are planned $O=($ on, off $)$. They cover the domain $O=[0,1]$ and verify $\forall o \in O$ :

$$
\left\{\begin{array}{l}
\mu \text { off } \eta^{(o)+\mu} \text { on } \eta^{(o)=1} \\
\mu_{\text {off } r_{b}}^{(o)+\mu_{\text {on } r_{b}}(o)=1} \\
\mu_{\text {off } \eta_{b b}}^{(o)+\mu} \text { on } \eta_{b b}(o)=1
\end{array}\right.
$$

where the switching controls given to relays are provided by the membership functions corresponding to $r_{l}, r_{b}, r_{l b}$ respectively, evaluated at $o$.

Based on this structure, the fuzzy rules for the relays' switching time are classified according to three intervals of dod:

$\operatorname{dod} \in X=\left[0, d_{d L_{\max }}\right]:$ the panels and/ or the battery bank supply the pump, $\operatorname{dod} \in Y=\left[d_{d L_{\text {min }}}, d_{d M_{\text {max }}}\right]$ : supplying the pump is preferred than charging the battery,

$\operatorname{dod} \in Z=\left[d_{d M_{\text {min }}}, d_{d L_{\text {max }}}\right]$ : charging the battery bank is preferred to supplying the pump when the panel produces insufficient power to the pump.

\subsubsection{Fuzzification}

Photovoltaic power $P_{p v}$

The membership functions of $\mu_{L}\left(x_{0 i}\right), \mu_{M}\left(x_{0 i}\right), \mu_{H}\left(x_{0 i}\right)$ corresponding to $P_{p v}$ are expressed as follows:

$$
\mu_{L}\left(x_{0 i}\right)=\left\{\begin{array}{ccc}
1 & \text { if } & 0<x<x_{L_{\min }} \\
\frac{x_{0 i}-x}{\varepsilon_{x_{0 i}}} & \text { if } & x_{L_{\min }}<x<x_{L_{\max }} \\
0 & & \text { otherwise }
\end{array}\right.
$$

$$
\begin{array}{r}
\mu_{M}\left(x_{0 i}\right)=\left\{\begin{array}{ccc}
\frac{x-x_{0 i}}{\varepsilon_{x_{0 i}}} & \text { if } & x_{M_{\min 1}}<x<x_{M_{\min 2}} \\
1 & \text { if } & x_{M_{\min 2}}<x<x_{M_{\max 1}} \\
\frac{x_{0 i}-x}{\varepsilon_{x_{0 i}}} & \text { if } & x_{M_{\max 1}}<x<x_{M_{\max 2}} \\
0 & & \text { otherwise }
\end{array}\right. \\
\mu_{H}\left(x_{0 i}\right)=\left\{\begin{array}{ccc}
1 & \text { if } & x>x_{H_{\max }} \\
\frac{x-x_{0 i}}{\varepsilon_{x_{0 i}}} & \text { if } & x_{H_{\min }}<x<x_{H_{\max }} \\
0 & & \text { otherwise }
\end{array}\right.
\end{array}
$$

Battery depth of discharge dod

The membership functions of $\mu_{d L}\left(d_{0 k}\right), \mu_{d M}\left(d_{0 k}\right), \mu_{d H}\left(d_{0 k}\right)$ corresponding to $\mathrm{dod}$ are expressed as follows:

$$
\mu_{d L}\left(d_{0 k}\right)=\left\{\begin{array}{ccc}
1 & \text { if } & 0<d<d_{d L_{\min }} \\
\frac{d_{0 k}-d}{\varepsilon_{d_{0 k}}} & \text { if } & d_{d L_{\min }}<d<d_{d L_{\max }} \\
0 & & \text { otherwise }
\end{array}\right.
$$




$$
\begin{gathered}
\mu_{d M}\left(d_{0 k}\right)=\left\{\begin{array}{ccc}
\frac{d-d_{0 k}}{\varepsilon_{d_{0 k}}} & \text { if } & d_{d M_{\min 1}}<d<d_{d M_{\min 2} 2} \\
1 & \text { if } & d_{d M_{\min 2}}<d<d_{d M_{\max 1}} \\
\frac{d_{0 k}-d}{\varepsilon_{d_{0 k}}} & \text { if } & d_{d M_{\max 1}}<d<d_{d M_{\max 2}} \\
0 & \text { otherwise }
\end{array}\right. \\
\mu_{d H}\left(d_{0 k}\right)=\left\{\begin{array}{ccc}
1 & \text { if } & d>d_{d H_{\max }} \\
\frac{d-d_{0 k}}{\varepsilon_{d_{0 k}}} & \text { if } & d_{d H_{\min }}<d<d_{d H_{\max }} \\
0 & & \text { otherwise }
\end{array}\right.
\end{gathered}
$$

\section{Water volume $v$}

The membership functions of $\mu_{v L}\left(v_{0 j}\right), \mu_{v M}\left(v_{0 j}\right), \mu_{v H}\left(v_{0 j}\right)$ corresponding to the water volume $v$ are expressed as follows:

$$
\begin{gathered}
\mu_{v L}\left(v_{0 j}\right)=\left\{\begin{array}{ccc}
1 & \text { if } & 0<v<v_{v L_{\min }} \\
\frac{v_{0 j}-v}{\varepsilon_{v_{0 j}}} & \text { if } & v_{v L_{\min }}<v<v_{v L_{\max }} \\
0 & \text { otherwise }
\end{array}\right. \\
\mu_{v M}\left(v_{0 j}\right)=\left\{\begin{array}{ccc}
\frac{v-v_{0 j}}{\varepsilon_{v_{0 j}}} & \text { if } & v_{v M_{\min 1}}<v<v_{v M_{\min 2} 2} \\
\frac{1}{v_{0 j}-v} & \text { if } & v_{v M_{\min 2}}<v<v_{v M_{\max 1}}
\end{array}\right. \\
\mu_{v H}\left(v_{0 j}\right)=\left\{\begin{array}{ccc}
\varepsilon_{v_{0 j}} & \text { if } & v_{v M_{\max 1}}<v<v_{v M_{\max 2}} \\
0 & & \text { otherwise } \\
\frac{1}{v-v_{0 j}} & \text { if } & v>v_{v H_{\max }} \\
\varepsilon_{v_{0 j}} & \text { if } & v_{v H_{\min }}<v<v_{v H_{\max }} \\
0 & & \text { otherwise }
\end{array}\right.
\end{gathered}
$$

\section{Power difference $\Delta P$}

The membership functions of $\mu_{f L}\left(f_{0 e}\right), \mu_{f H}\left(f_{0 e}\right)$ corresponding to $\Delta P$ are expressed as follows:

$$
\begin{gathered}
\mu_{f L}\left(f_{0 e}\right)=\left\{\begin{array}{ccc}
1 & \text { if } & 0<f<f_{f L_{\min }} \\
\frac{f_{0 e}-f}{\varepsilon_{f_{0 e}}} & \text { if } & f_{f L_{\text {min }}}<f<f_{f L_{\text {max }}} \\
0 & \text { otherwise }
\end{array}\right. \\
\mu_{f H}\left(f_{0 e}\right)=\left\{\begin{array}{ccc}
1 & \text { if } & f>f_{f H_{\max }} \\
\frac{f-f_{0 e}}{\varepsilon_{f_{0 e}}} & \text { if } & f_{f H_{\text {min }}}<f<f_{f H_{\text {max }}} \\
0 & \text { otherwise }
\end{array}\right.
\end{gathered}
$$

Switching control of the relays $R_{b}, R_{l}, R_{l b}$

The relay membership functions $\mu_{\text {off } r_{i}, r_{b}, r_{l b}}\left(o_{0 z}\right)$ and $\mu_{o n} r_{l}, r_{b}, r_{b b}\left(o_{0 z}\right)$ corresponding to the relays $R_{b}, R_{l}, R_{l b}$ are expressed as follows:

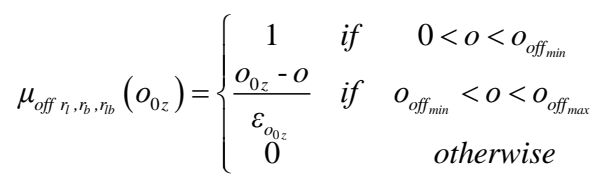

$$
\mu_{o n r_{i}, r_{b}, r_{i b}}\left(o_{0 z}\right)=\left\{\begin{array}{ccc}
1 & \text { if } & o>o_{o n_{\max }} \\
\frac{o-o_{0 z}}{\varepsilon_{o_{0 z}}} & \text { if } & o_{o n_{\min }}<o<o_{o n_{\max }} \\
0 & & \text { otherwise }
\end{array}\right.
$$

\subsubsection{Inference Diagram}

Based on the fuzzified inputs, the rules set serve to decide the relays' switching control, which are deduced using the modes explained previously. The control signals of the relays are given following this equation:

$$
r_{0 l, b, l b}=\frac{\int_{0}^{1} r_{o n} \mu_{r_{\text {on }}} d r_{o n}}{\int_{0}^{1} \mu_{r_{\text {on }}} d r_{o n}}
$$

\subsubsection{Defuzzification}

The control of the three relays is deduced by (Fig. 4):

$$
\begin{aligned}
& \text { If } r_{l, b, l b}<0.5 \text { then } R_{l, b, l b} \text { is off } \\
& \text { If } r_{l, b, l b}>0.5 \text { then } R_{l, b, l b} \text { is on }
\end{aligned}
$$

\section{RESULTS AND DISCUSSION}

In order to test the proposed algorithm efficiency, the algorithm is experimentally validated using measured climatic data of a land planted with tomatoes, located in the Northern of Tunisia ((latitude: $36.64^{\circ}$, longitude: $9.60^{\circ}$ ). This application is prompted by the fact that tomatoes must be irrigated regularly, especially during flowering and fruit formation [26]. The irrigation is gravity-based: $200 \mathrm{~m}^{3} / \mathrm{h}$ just before sunrise, to irrigate a 10 ha field by a low-pressure gravity-driven drip system.

\subsection{Algorithm Parameterization}

\subsubsection{Photovoltaic Power $P_{p}$}

The photovoltaic power $P_{p v}$ is classified as follows:

$$
\begin{aligned}
& \text { If } P_{p v} \in\left[\begin{array}{ll}
0 & 10
\end{array}\right] \text { then } P_{p v} \text { is considered low } \\
& \text { If } P_{p v} \in\left[\begin{array}{ll}
10 & 4500
\end{array}\right] \text { then } P_{p v} \text { is considered medium } \\
& \text { If } P_{p v} \in\left[\begin{array}{ll}
4500 & 10000
\end{array}\right] \text { then } P_{p v} \text { is considered high } \\
& \text { 3.1.2 }
\end{aligned}
$$

The battery' non-linear model detailed in $[13,21]$ is used here to evaluate the $\mathrm{dod}$, which is classified as follows:

$$
\begin{aligned}
& \text { If dod } \in\left[\begin{array}{ll}
0 & 0.02
\end{array}\right] \text { then dod is considered low. } \\
& \text { If dod } \in\left[\begin{array}{ll}
0.02 & 0.9
\end{array}\right] \text { then dod is considered medium. } \\
& \text { If dod } \in\left[\begin{array}{ll}
0.9 & 1
\end{array}\right] \text { then dod is considered high. }
\end{aligned}
$$

\subsubsection{Stored Water Volume v}

Using the water need model $[24,26]$, the water volume $V$ corresponding to each month of tomatoes' vegetative cycle at the target location is:

The mean water volume of March $\left(m_{1}\right)$ is $l_{1}=60 \mathrm{~m}^{3} /$ day.

The mean water volume of April $\left(m_{2}\right)$ is $l_{2}=100$ $m^{3} /$ day.

The mean water volume of May $\left(m_{3}\right)$ is $l_{3}=179 \mathrm{~m}^{3} /$ day. 
The mean water volume of June $\left(m_{4}\right)$ is $l_{4}=241 \mathrm{~m}^{3} /$ day.

The mean water volume of July $\left(m_{5}\right)$ is $l_{5}=321 \mathrm{~m}^{3} /$ day.

The fuzzification of the water volume depends on the month and is described in Table 1 .

\subsection{Experimental validation and discussion}

A scale plant installed in the laboratory in the Engineering Systems and Automatic department of the University of Valladolid has been used. The plant is composed of a pump, a $0.05 \mathrm{~m}^{3}$ reservoir, a $150 \mathrm{~W}$ Programmable Power Supply, which is used as photovoltaic panel associated to an MPPT bloc, and a 12A.h/12V lead-acid battery. The fuzzy algorithm decides the switching of the relays that link the PPS, the battery and the pump. The simulation' results of the fuzzy management algorithm are presented in Fig. 4. The experimental results are presented in Fig.5 and Fig.6.

In fact, in Fig.4, during the morning, both the battery bank and the panels supply the pump, since the battery bank is charged. This corresponds to (mode2). During the day, when there is an energy excess, it is used to charge the battery bank by switching on the relays $R_{l}$ and $R_{b}$ (mode 3 ). Mode 5 is possible when the battery bank is full and the panel produces just $110 \%$ of the power needed by the pump. In this case, only the relay $R_{l}$ is switched on. At sunset, the $\operatorname{dod}$ is maintained below 0.9 and the reservoir is full (mode 1).

The experimental results, illustrated in Fig. 5, show that the algorithm allows the pump to be supplied and the battery' bank safe operation to be ensured thanks to the control' switching of the relays $R_{b}, R_{l}, R_{l b}$.In fact, in Fig.5, the case when the panel produces energy in excess (case 1) is presented. In this case, two operation modes are possible: in mode 3 the relays $R_{l}$ and $R_{b}$ are switched on since the reservoir is medium and the battery bank' dod is medium. When the reservoir is full, the relay $R_{l}$ is switched off and all the energy generated from the PPS is used to charge the battery' bank (mode 4).

When the panel produces unsufficient power to the pump' operation (case 2) (Fig.6), the relay $R_{l b}$ is switched on and thus, the battery bank supply also the pump. In this case, the relays $R_{l b}$ and $R_{l}$ are switched on (mode 2$)$.

Hence, Fuzzy logic allows the optimization of the energy generated to be ensured, the water volume needed by the crops to be pumped and ta safe battery bank' operating.
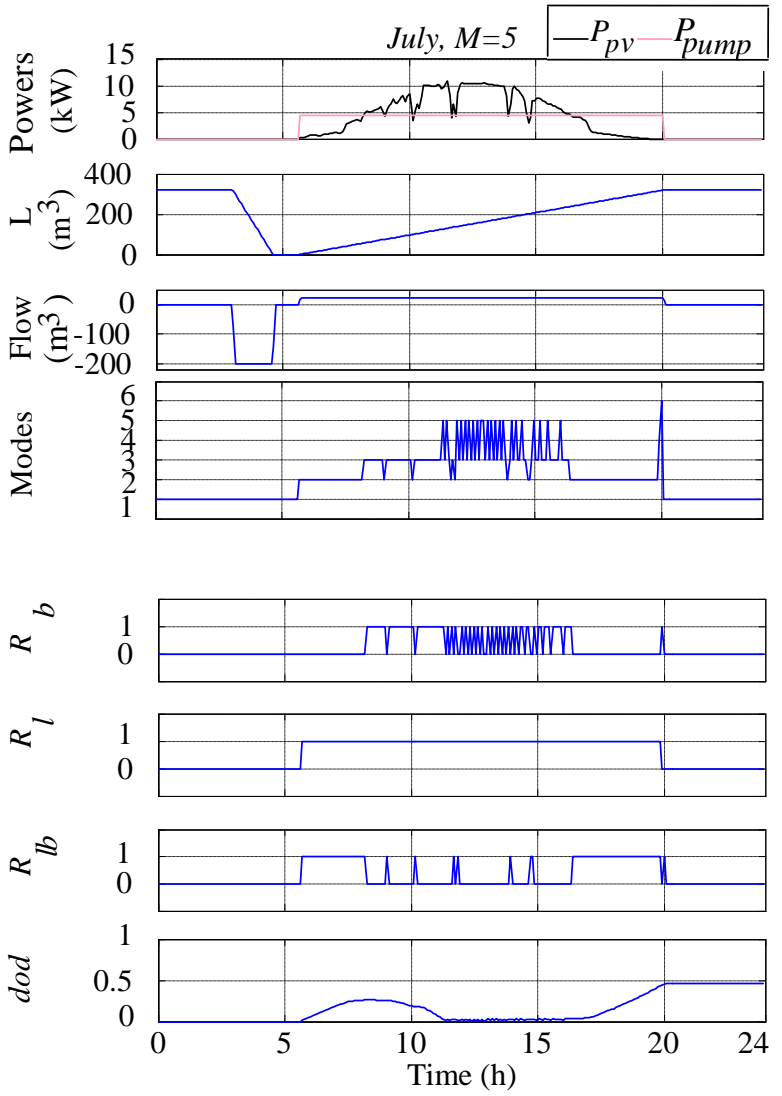

Fig. 4. Fuzzy energy management results for a typical day in July
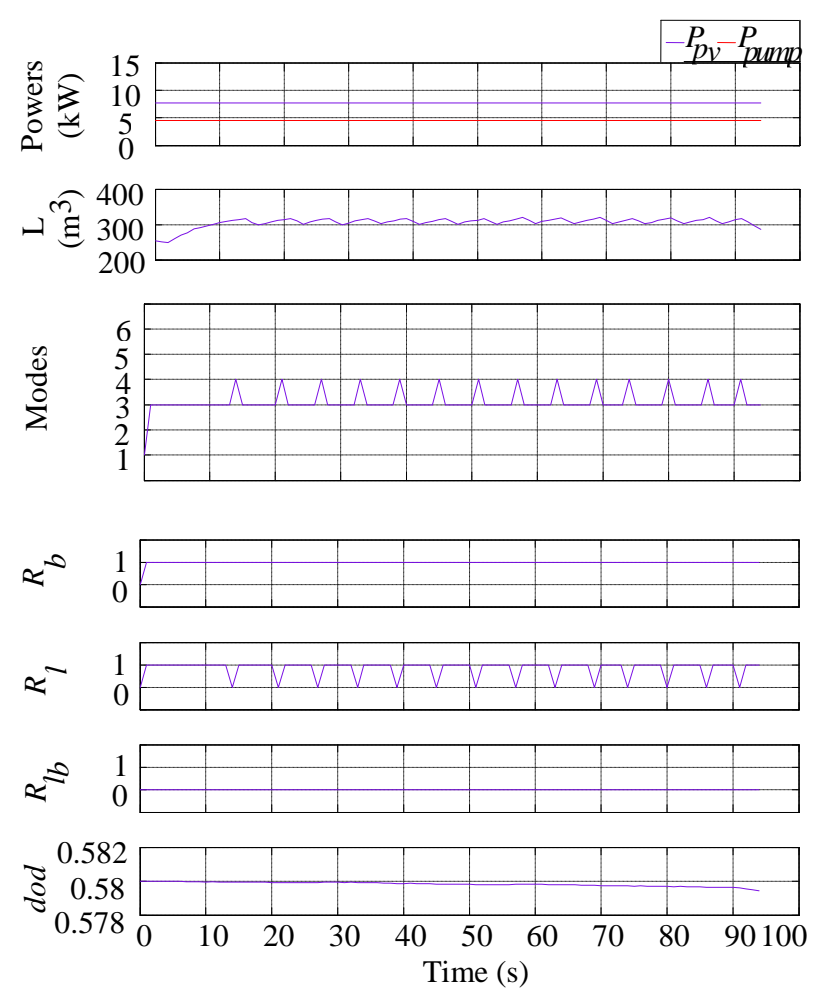

Fig. 5. Experimental results for the Fuzzy algorithm in July (case 1) 
Table 1. Water fuzzification corresponding to each month $M$

\begin{tabular}{|l|c|c|c|c|c|}
\hline & $m_{1}:$ March & $m_{2}:$ April & $m_{3}:$ May & $m_{4}:$ June & $m_{5}:$ July \\
\hline$l_{1}$ & low & low & low & low & low \\
\hline$l_{2}$ & high & medium & low & low & low \\
\hline$l_{3}$ & high & high & medium & low & low \\
\hline$l_{4}$ & high & high & high & medium & low \\
\hline$l_{5}$ & high & high & high & high & medium \\
\hline
\end{tabular}

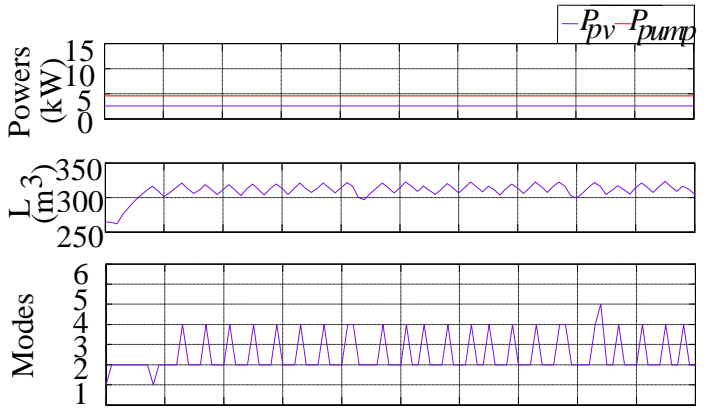

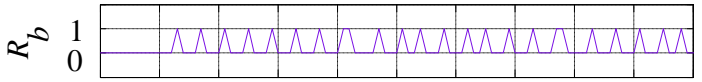
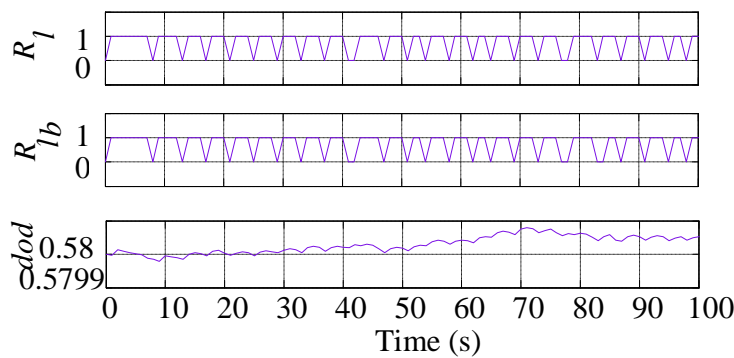

Fig. 5. Experimental results for the Fuzzy algorithm in July (case 2)

\section{CONCLUSION}

A fuzzy algorithm for the energy management of a photovoltaic irrigation installation composed of photovoltaic panels, a lead-acid battery bank and a submerged pump, has been presented and experimentally validated. The algorithm makes decisions on the interconnection time of the photovoltaic panel, the battery bank and the pump, by taking into account some constraints related with the photovoltaic power generated, the battery depth of discharge and the amount of stored water.

The algorithm has been tested in a 1:215 pilot system for a specific case study (tomatoes' irrigation) during the most critical month for the crops (July), with photovoltaic energy produced by a controllable power supply, a lead- acid battery bank and a water pump. Using data from the target location, the experimental results show that the algorithm ensures pumping the water' volume needed by tomatoes, the system autonomy and the battery bank' safety. It must be pointed out that the proposed algorithm is general, in the sense that it can be used for PV irrigation of systems of different sizes, by providing the monthly water demand.

As a general conclusion, we have shown the efficiency of fuzzy tool in the energy management for water pumping installation, and that a simple energy management system can improve the operation of off-grid PV systems.

\section{ACKNOWLEDGMENTS}

The authors would like to thank Mr Lamine Yahyaoui, from the Tunisian Ministry of Agriculture, for providing us with data and $\mathrm{Mr}$ Graciano Garcia from the University of Valladolid, Spain for his contribution. The authors would like to acknowledge the support of the Engineering of Systems and Automatic of Valladolid (ISA). Miss Yahyaoui is supported by a grant from MICInn BES-2011-047807.

\section{REFERENCES}

[1] Al-Alawi, A., Al-Alawi, SM., Islam, SM. 2007. Predictive control of an integrated PV-diesel water and power supply system using an artificial neural network. Renew Energy. (2007), 1426-39.

[2] Kuznia, L., Zeng, B., Centeno, G., Miao, Z. 2012. Stochastic optimization for power system configuration with renewable energy in remote areas. Ann Oper Res (2012), 1-22.

[3] European Commission. IEA-AIE. 2013. "Key World Energy Statistics 2013", International Energy Agency, www.iea.org, Rapport 2013.

[4] Kaikhurst, ET. 1998. Village electrification an evaluation of options in remote area systems. The World Directory of Renewable Energy Suppliers and Services. (1998).

[5] Bouzidi, B., Malek, A., \& Haddadi, M. 2006. Rentabilité économique des systèmes de pompage photovoltaïques. Revue des Energies Renouvelables. (2006), 187 - 197.

[6] Kumar, A., \& Kandpal, T. C. 2007. Renewable energy technologies for irrigation water pumping in India: a preliminary attempt towards potential estimation. Energy. (2007), 861-870.

[7] Gopal, C., Mohanraj, M., Chandramohan, P., \& Chandrasekar, P. 2013. Renewable energy source water pumping systems - A literature review. Renewable and Sustainable Energy Reviews. (2013), 351-370.

[8] Hmidet, A., Gammoudi, R., Hasnaoui, O., \& Dhifaoui, R. 2014. Analog MPPT controller circuit used in photovoltaic pumping systems. In Renewable Energy Congress (IREC), 2014 5th International, 1-6. IEEE. 
[9] Yahyaoui, I., Chaabene, M., \& Tadeo, F. 2013. An algorithm for sizing photovoltaic pumping systems for tomatoes irrigation. In Proceedings of the International Conference on Renewable Energy Research and Applications (ICRERA), 2013, 1089-1095. IEEE.

[10] Ma, T., Yang, H., Lu, L., \& Peng, J. 2014. Pumped storage-based standalone photovoltaic power generation system: modelling and techno-economic optimization. Applied Energy. (2014), 649-659.

[11] Manolakos, D., Papadakis, G., Papantonis, D, Kyritsis, S.2004. A stand-alone photovoltaic power system for remote villages using pumped water energy storage. Energy. (2004), 57-69.

[12] Arrouf, M., Ghabrour. S. 2007. Modelling and simulation of a pumping system fed by photovoltaic generator within the Matlab/Simulink programming environment. Desalination. (2007), 23-30.

[13] Sallem, S., Chaabene, M., \& Kamoun, M.B.A. 2009. Optimum energy management of a photovoltaic water pumping system. Energy Conversion and Management. (2009), 2728-2731.

[14] Ben Salah, C., Chaabene, M., \& Ben Ammar, M. 2008. Multi-criteria fuzzy algorithm for energy management of a domestic photovoltaic panel. Renewable Energy. (2008), 993-1001.

[15] Yahyaoui, I., Sallem, S., Kamoun, M. B A., \& Tadeo, F. 2014. A proposal for management of off-grid photovoltaic systems with non-controllable loads using fuzzy logic. Energy Conversion and Management. (2014), 835-842.

[16] Berrazouane, S., \& Mohammedi, K. 2014. Parameter optimization via cuckoo optimization algorithm of fuzzy controller for energy management of a hybrid power system. Energy Conversion and Management. (2014). 652-660.

[17] Welch, R. L., \& Venayagamoorthy, G. K. 2010. Energy dispatch fuzzy controller for a grid-independent photovoltaic system. Energy Conversion and Management. (2010), 928-937.

[18] Mousa, K., Croock, A., \& Abdullah, N, M. 2014. Fuzzy based Decision Support Model for Irrigation System Management. International Journal of Computer Applications. (2014), 14-20.

[19] Hahn, F. 2011. Fuzzy controller decreases tomato cracking in greenhouses. Computers and Electronics in Agriculture. (2011). 21-27.

[20] Cheikh, M. A., Aïssa, B. H., Malek, A., \& Becherif, Mohamed. 2010. Mise au point d'une régulation floue pour serre agricole à énergie solaire'. Revue des Energies Renouvelables. (2010), 421-443.
[21] Sallem, S., Chaabene, M., \& Kamoun, M. B A. 2009. Optimum energy management of a photovoltaic water pumping system. Energy Conversion and Management. (2009), 2728-2731.

[22] Kahraman, C., Kaya. I., \& Cebi, S. 2009. A comparative analysis for multiattribute selection among renewable energy alternatives using fuzzy axiomatic design and fuzzy analytic hierarchy process. Energy. (2009), 16031616.

[23] Hunaish, A, S., Radhi, J. 2014. PLC based Implementation of Fuzzy Controller for Boost Converter. International Journal of Computer Applications. (2014). 36- 44.

[24] Yahyaoui, I., Chaabene, M., \& Tadeo. 2015. Energy Management for Photovoltaic Irrigation with a Battery Bank, IJEOE, accepted (August, 2014).

[25] Sharma, B., Katiyar, V.K., Gupta, A.K. 2014. Fuzzy Logic Model for the Prediction of Traffic Volume in Week Day. (2014). International Journal of Computer Applications, 0975 - 8887.

[26] Shankara, Naika., De Jeude, Joep Van Lidt., De Goffau, Marja., Hilmi, Martin; \& Van Dam, Barbara. 2005. Production, processing and marketing. Agromisa Foundation and CTA.

\section{AUTHORS' PROFILES}

Imene Yahyaoui is a $\mathrm{PhD}$ student at the Engineering Industrial School of Valladolid, Spain. Her interest is renewable energy applications and power electronics. She is a member in international congress organization committee, namely the International Congress of Renewable Energies (IREC) and the World Academy of Science, Engineering and Technology (WASET). She is a reviewer for Energy Conversion and Management and Renewable and Sustainable Energy journals.

Maher Chaabene is a Professor at University of Sfax, TUNISIA- in Electrical Engineering and Computer Science Department. His major field of study is Renewable Energy systems: measurements, sizing and management. He is guest editor for two special issues for Energy Conversion and Management Journal, author of 20 research papers and more than 50 conference papers.

Fernando Tadeo is a Professor at the School of Engineering, University of Valladolid since 2010. He graduated from the same university, in Physics in 1992, and in Electronic Engineering in 1994. Aftercompleting an M.Sc. in Control Engineering in the University of Bradford, U.K., he went back to Valladolid, where he got his Ph.D. degree. His main interest area is Advanced Process Control, focused on applications in Desalination and Renewable Energies (Wind, Solar and Osmotic). 